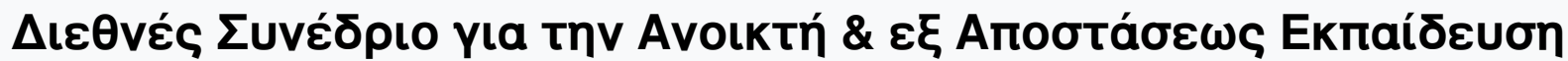

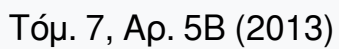

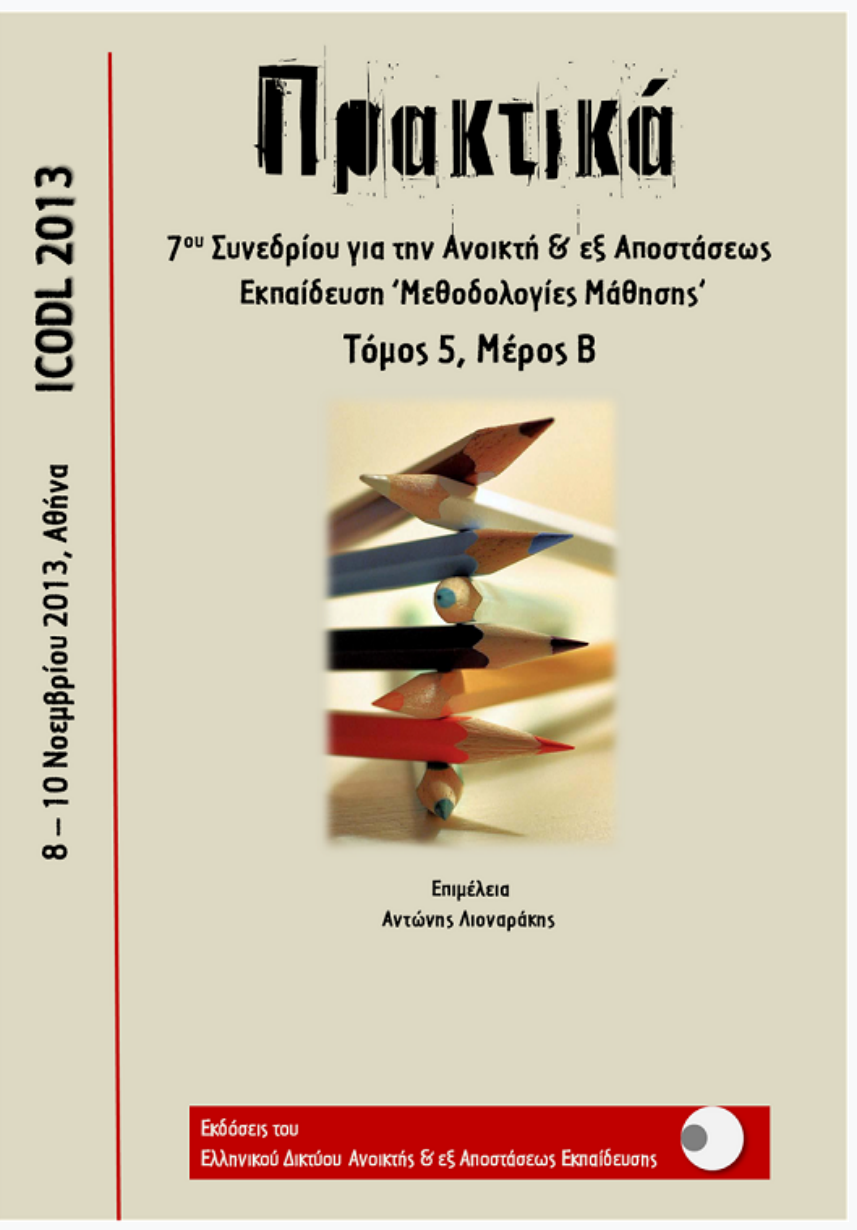

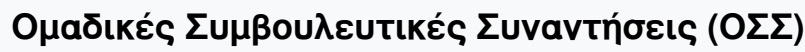

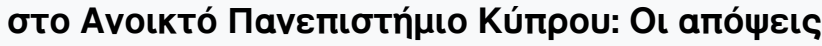

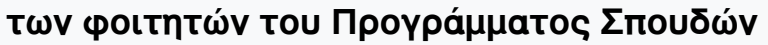

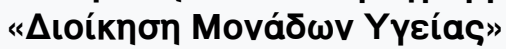

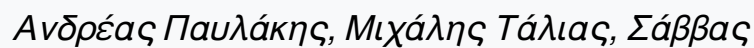
Zavvétos

doi: $\underline{10.12681 / \text { icodl. } 557}$ 


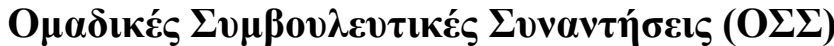

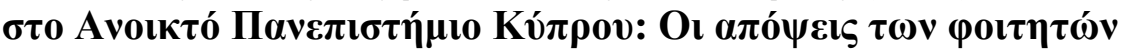

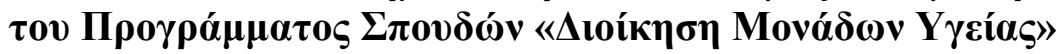

\section{Group Counseling Tutorials at Open University of Cyprus: Health Care Management Students' opinions}

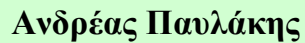

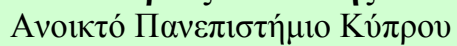

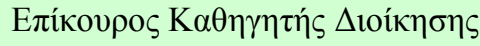

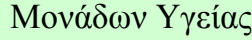 \\ a.pavlakis@ouc.ac.cy
}

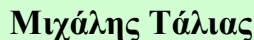

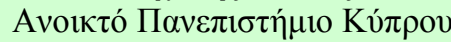

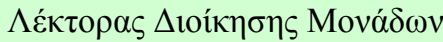 \\ Yүzías \\ m.talias@ouc.ac.cy
}

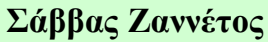

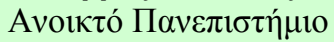

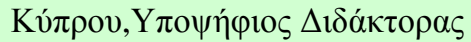

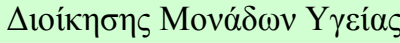 \\ s.zannetos@st.ouc.ac.cy
}

\begin{abstract}
Even though our University holds Group Counseling Tutorials (GCT) since its first day, up to now there hasn't been any research on how GCTs contribute to the educational process. Group Counseling Tutorials and, particularly, their content and the educational techniques utilized the role of the counseling professor and the emotional states of the students are the three subjects studied in this research. The achievement of this goal a specially-designed questionnaire was used, so as to record the organizational type, the techniques used in a GCT, the role of the counseling professor, as well as the students' perceived levels of stress.

Out of 181 students participating in the research, 159 are part of the "Healthcare Management" Programme. The educational needs of the students are covered in a large degree during the GCTs. However, some of the students believe that the content of the GCTs is structured according to their experiences while others state that GCT content is based on preexisting knowledge. Most of the participants believe that the counseling professors are guides, catalysts, friends, masters of their field, spirit uplifters, intermediators and partners.

At the same time, some of the participants wish, mainly, to have the counseling professor as a partner and guide. Mastery of field, meaning the counseling professor teaching conventionally and imparting knowledge, is rated last. Essentially, the research findings and the contextual statistical analyses show that, in a great degree, the participants' needs and demands are met and that the theoretical frame of distance learning for the education of adults is fully deployed.
\end{abstract}

Keywords: distant learning, group tutorials, adjunct professors' role, students' opinions

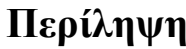

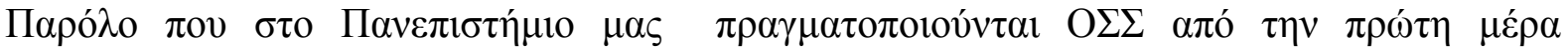

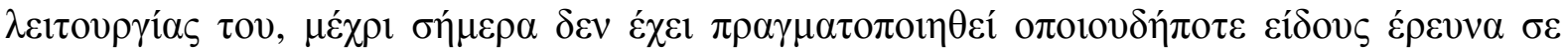

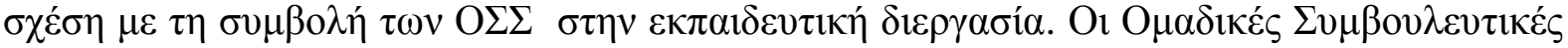

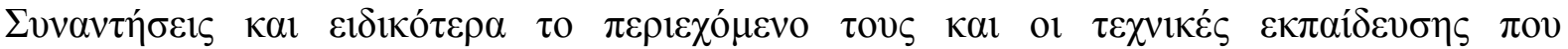

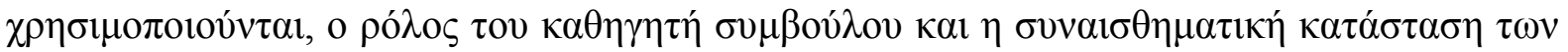

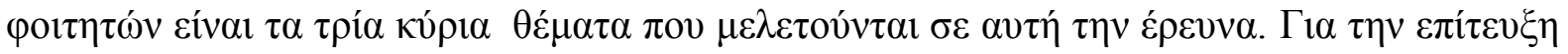

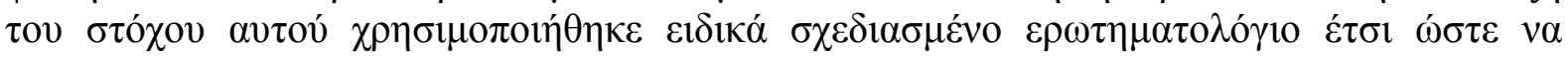

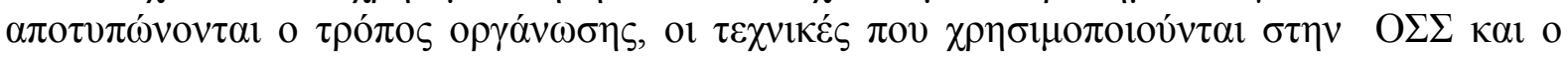

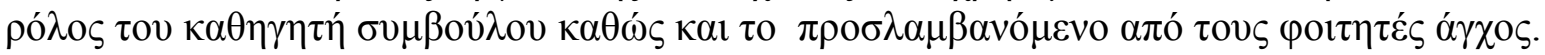




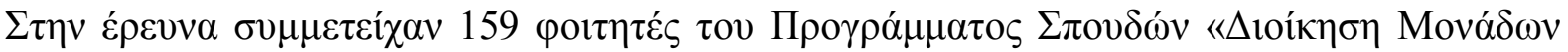

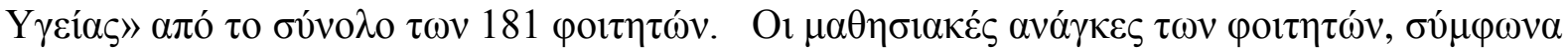

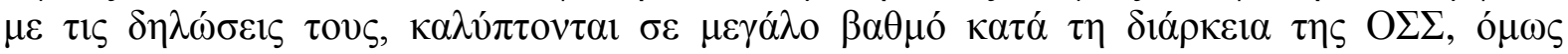

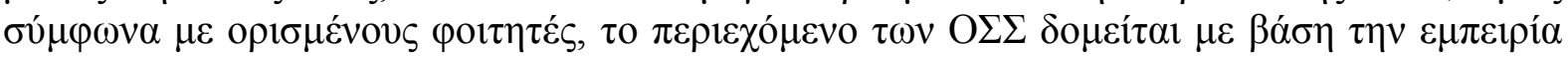

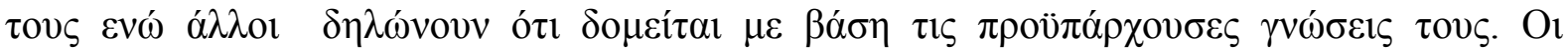

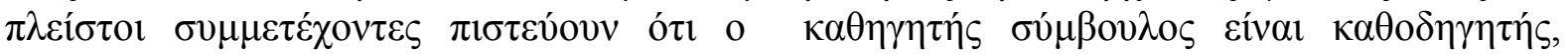

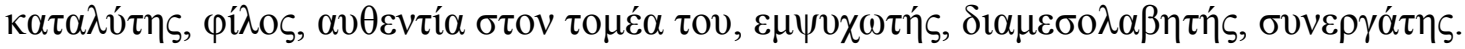

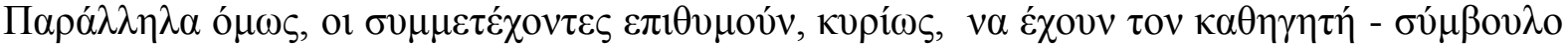

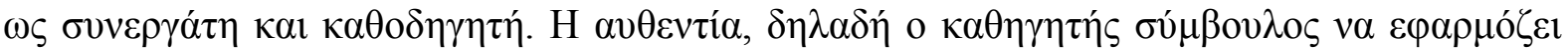

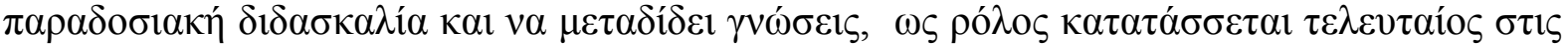

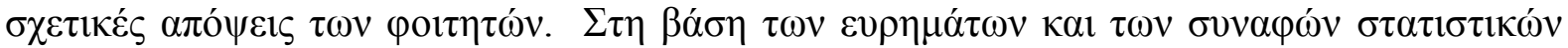

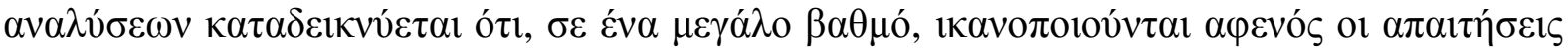

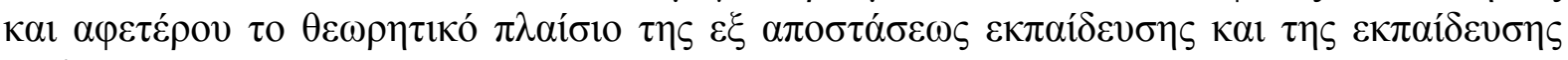
$\varepsilon v \eta \lambda i ́ \kappa \omega v$.

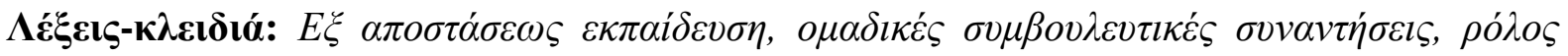
$\kappa \alpha \theta \eta \gamma \eta \tau \dot{\eta}-\sigma \nu \mu \beta o v ́ \lambda o v, \alpha \pi o ́ \psi \varepsilon \iota \varsigma \tau \omega \nu$

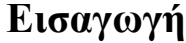

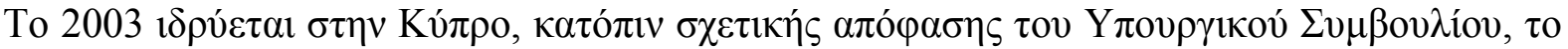

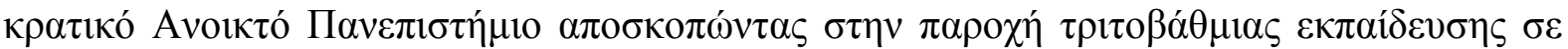

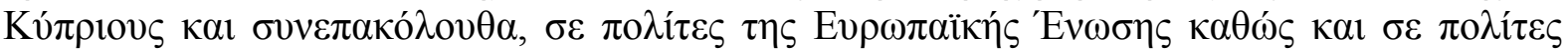

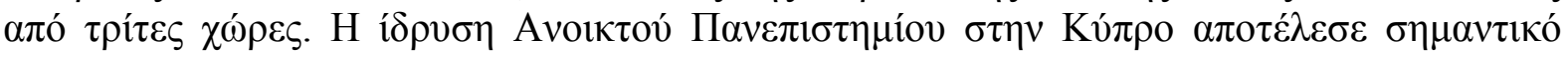

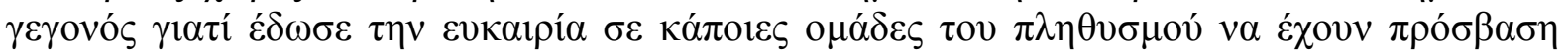

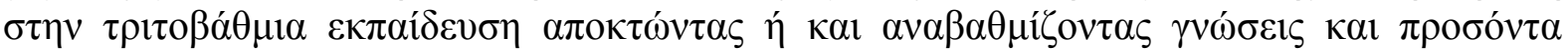

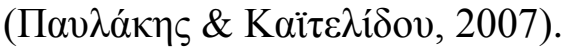

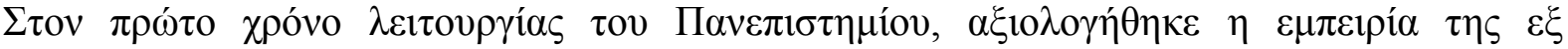

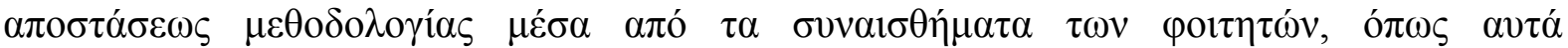

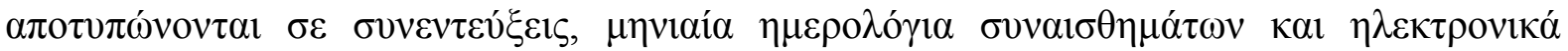

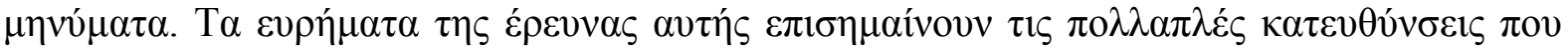

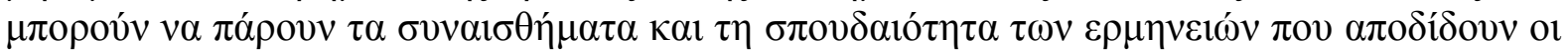

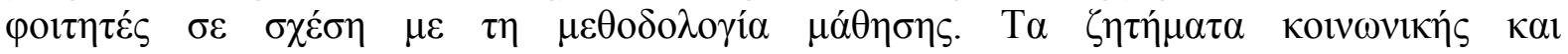

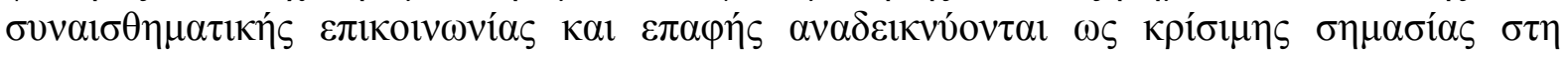

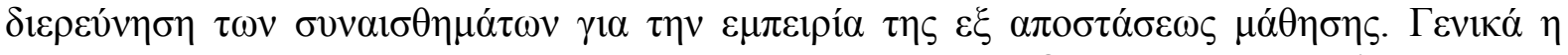

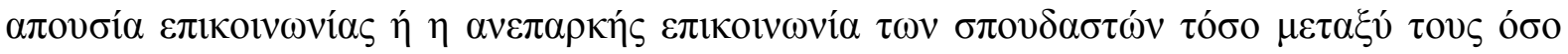

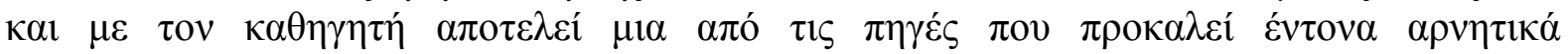
$\sigma v v \alpha 1 \sigma \theta \eta \dot{\mu} \mu \tau \alpha$ (Zembylas et al. 2008).

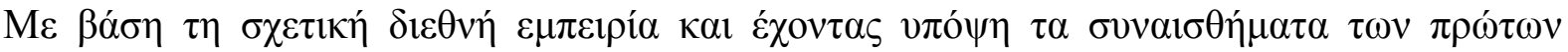

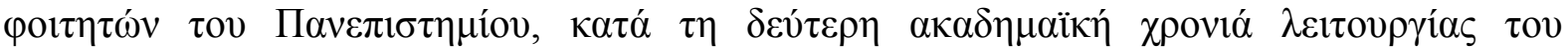

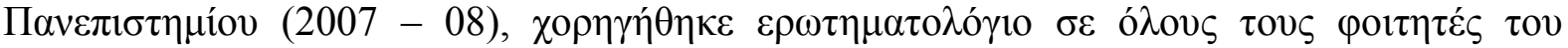

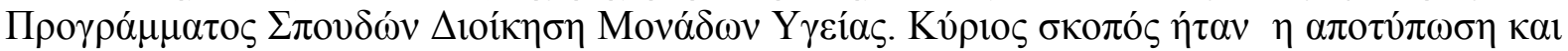

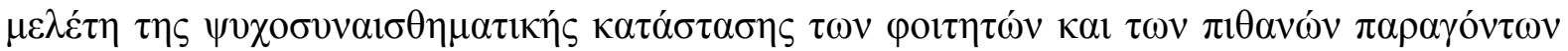
$\pi \mathrm{ov} \tau \eta \nu \varepsilon \pi \eta \rho \varepsilon \alpha ́ \zeta \zeta o v v$.

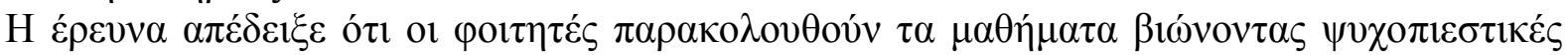

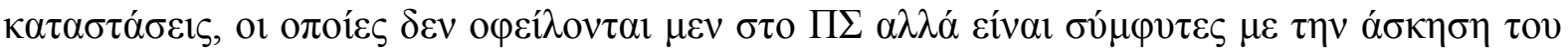

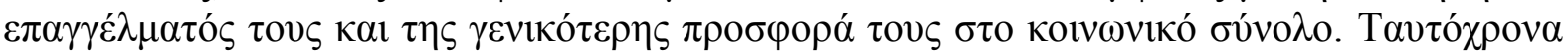

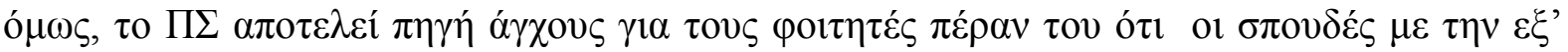

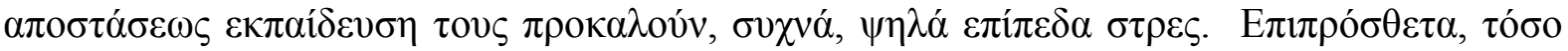




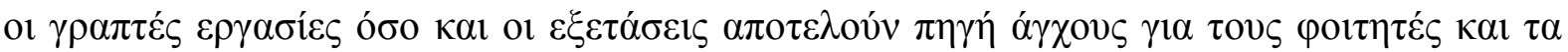

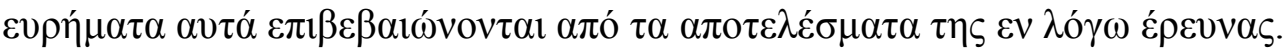

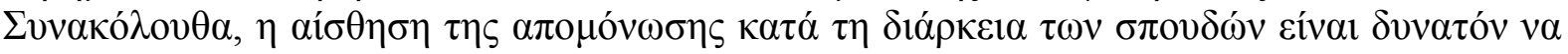

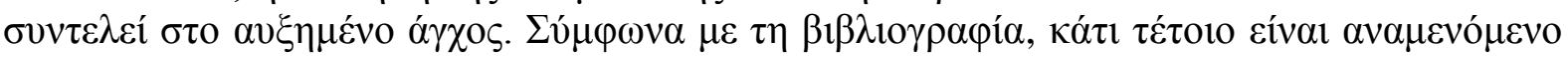

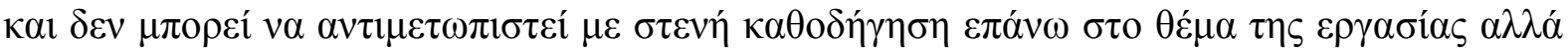

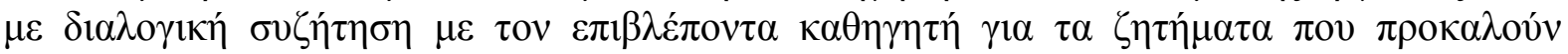
$\alpha \beta \varepsilon \beta \alpha$ ı́ $\tau \eta \tau \alpha$ (Pavlakis \& Kaitelidou, 2012).

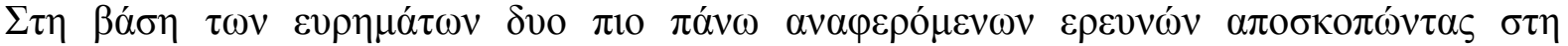

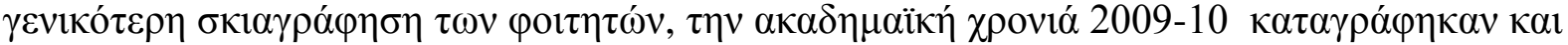

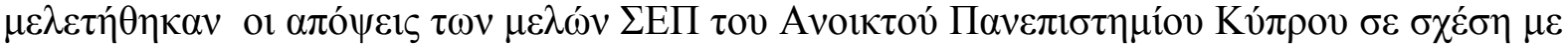

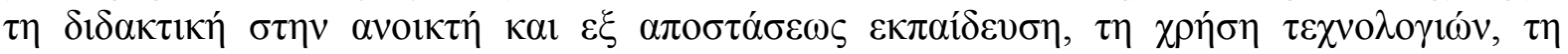

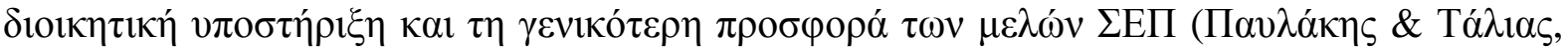

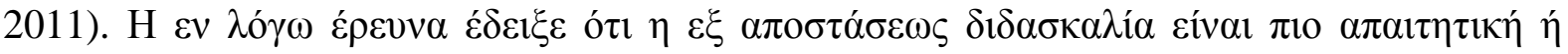

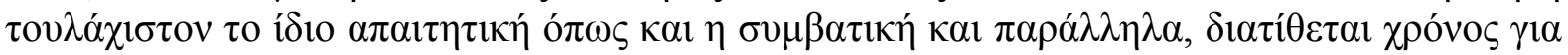
$\tau \eta \delta 1 \varepsilon \kappa \pi \varepsilon \rho \alpha i ́ \omega \sigma \eta \tau \omega v ~ \sigma v v \alpha \varphi \omega ́ v ~ v \pi 0 \chi \rho \varepsilon \omega ́ \sigma \varepsilon \omega v \tau \omega v \mu \varepsilon \lambda \omega ́ v \Sigma E \Pi$.

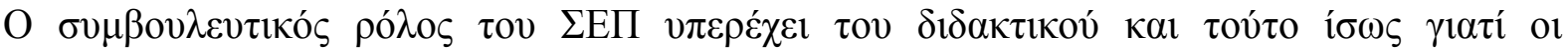

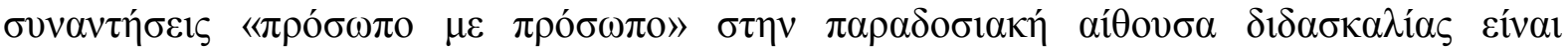

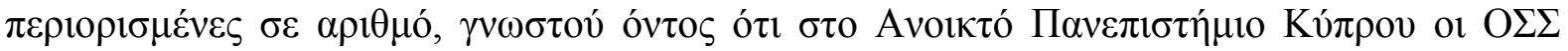

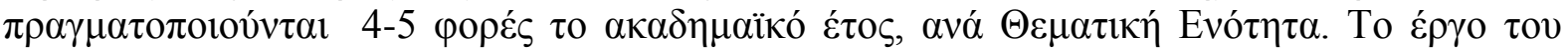

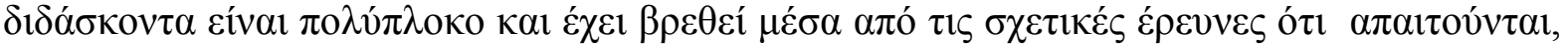

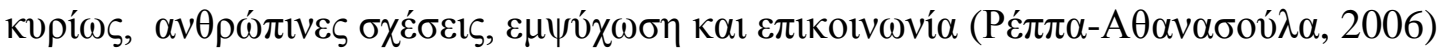

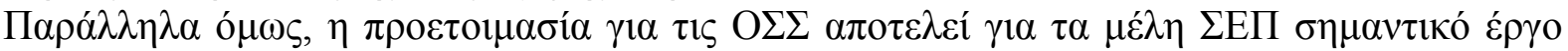

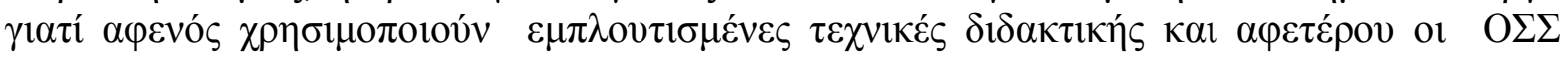

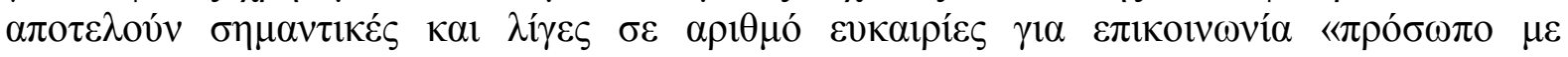

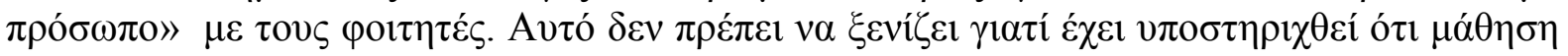

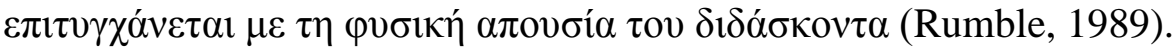

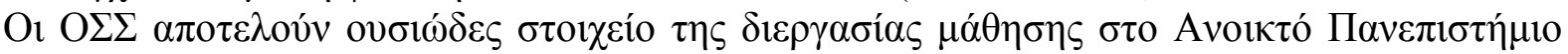

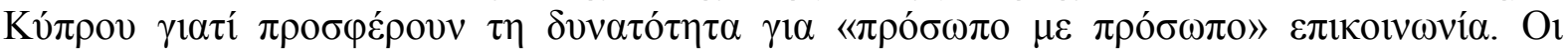

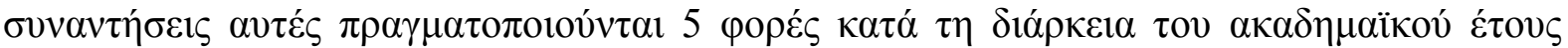

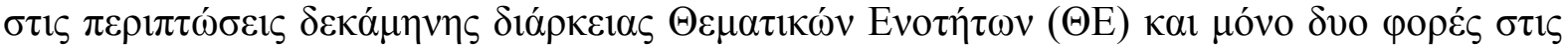

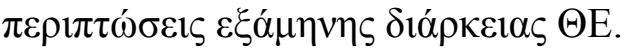

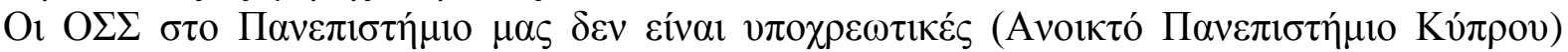

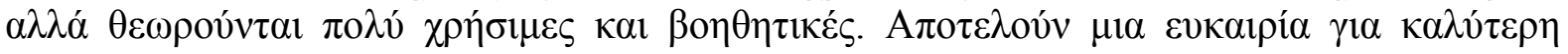

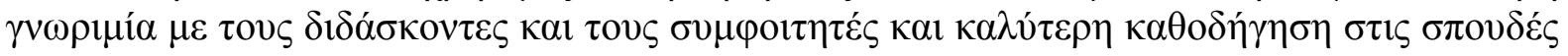

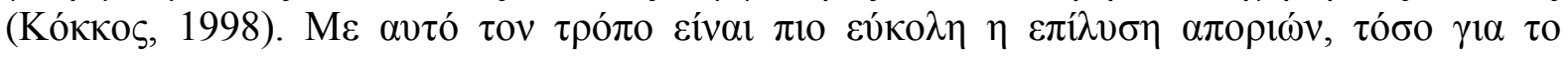

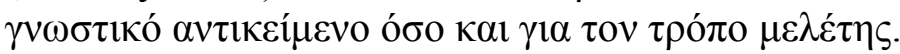

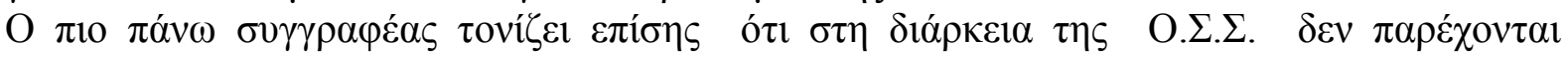

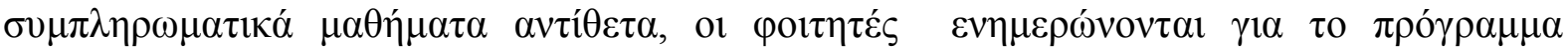

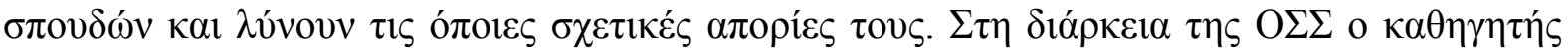

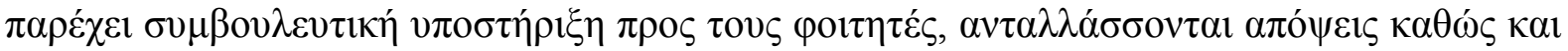

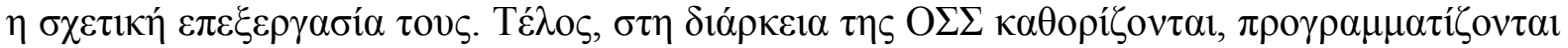

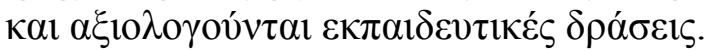

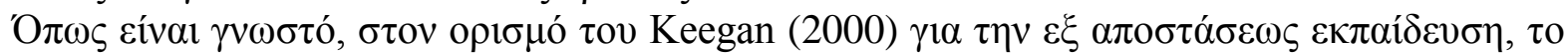

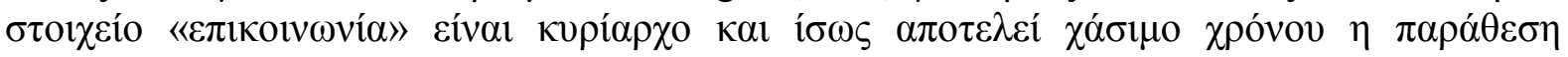

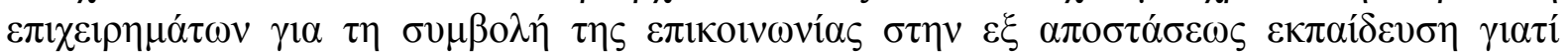

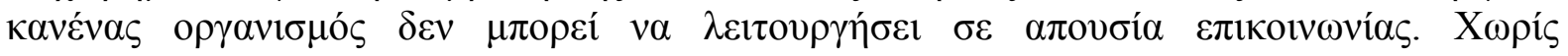

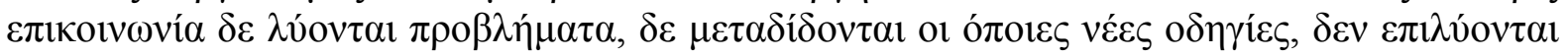

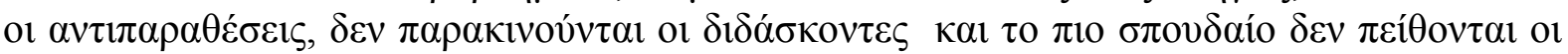

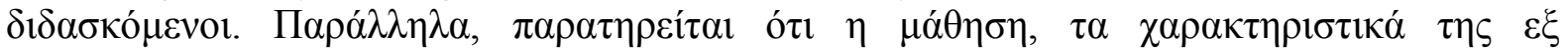




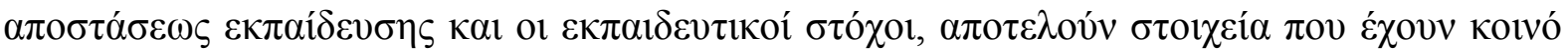

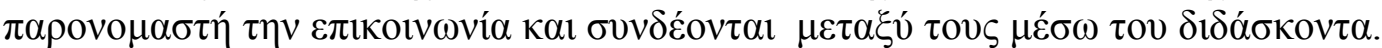

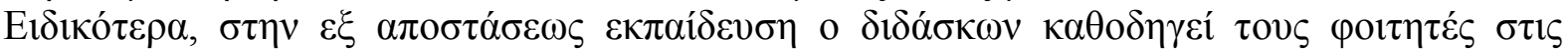

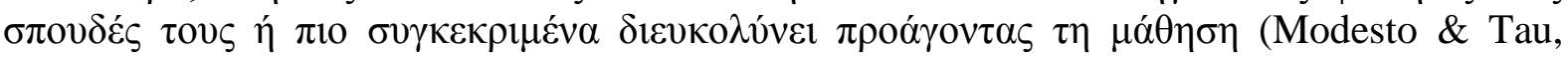

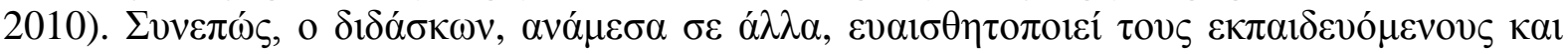

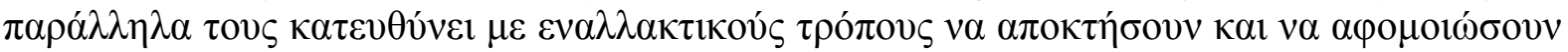

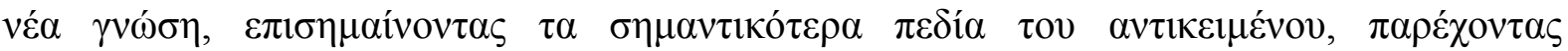

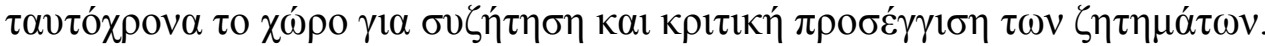

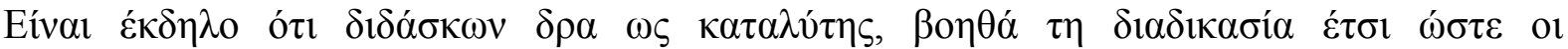

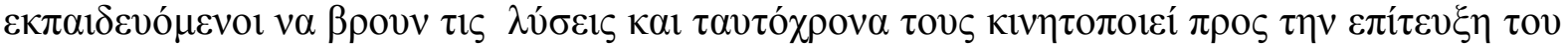

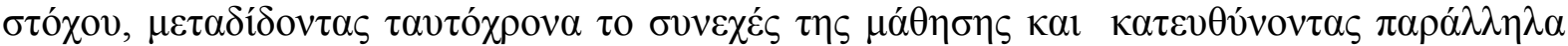

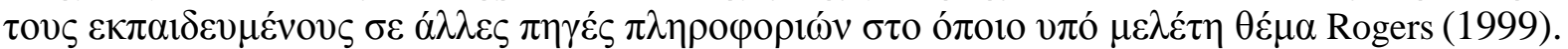

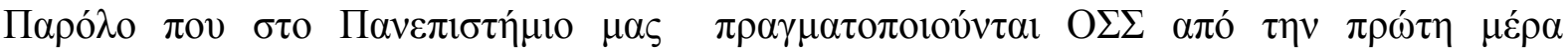

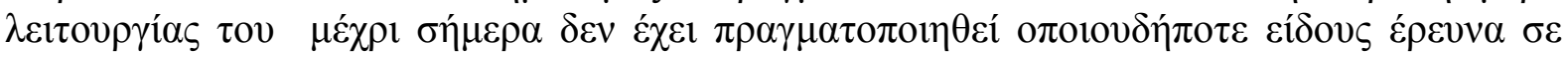

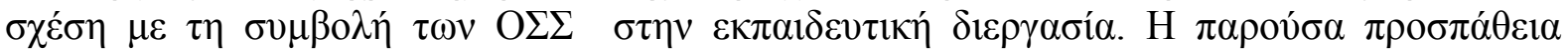

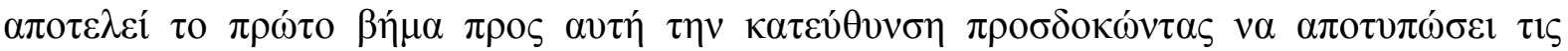

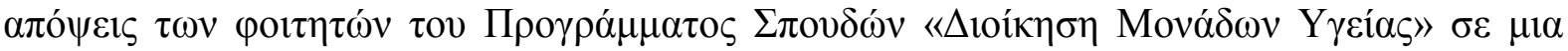

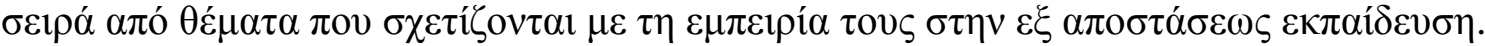

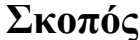

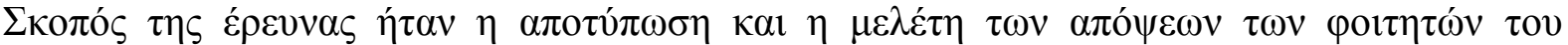

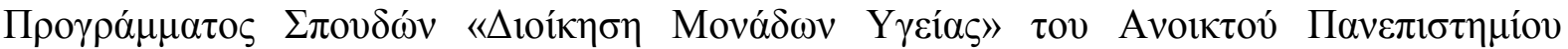

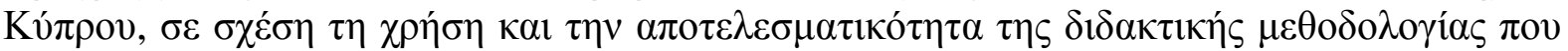

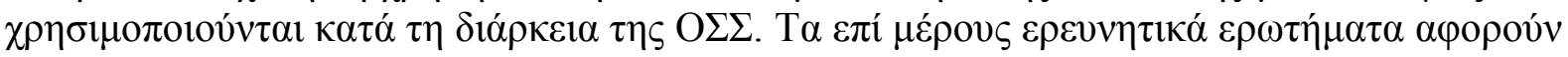

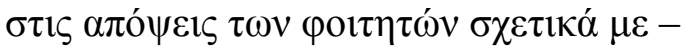

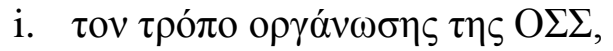

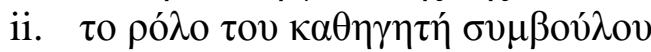

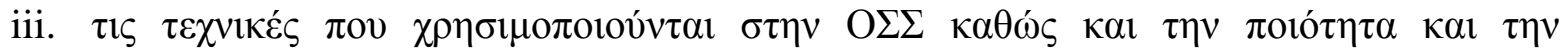

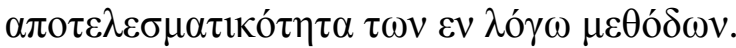

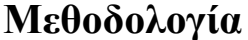

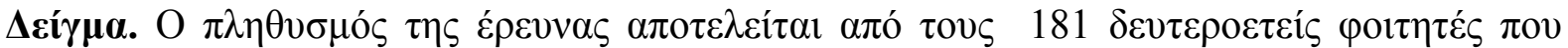

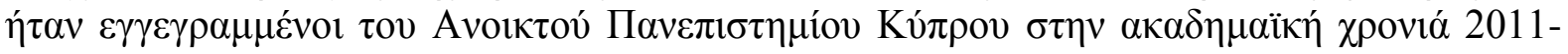

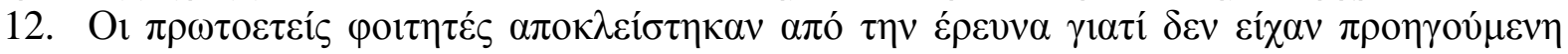

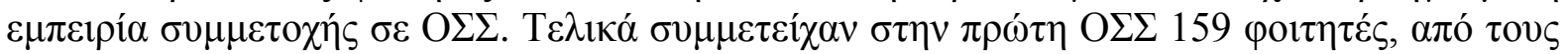

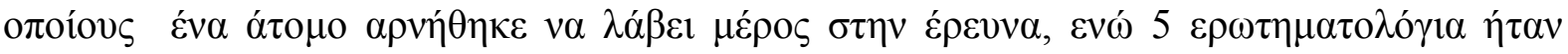

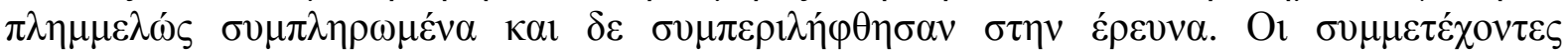

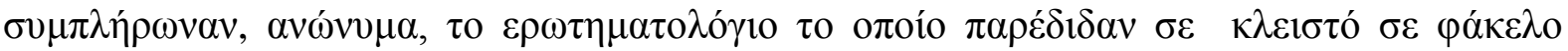

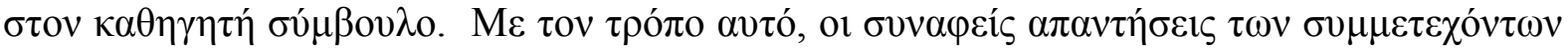

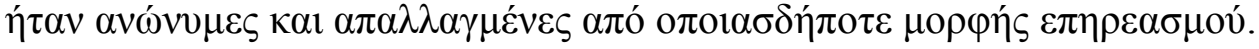

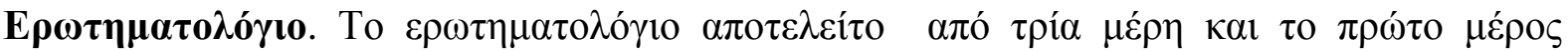

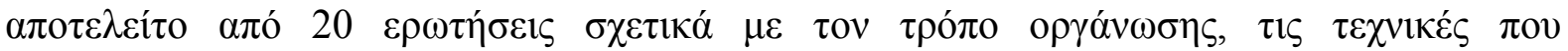

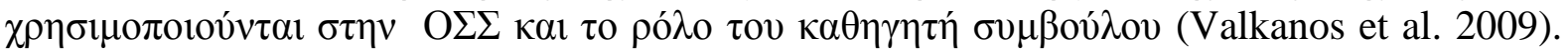

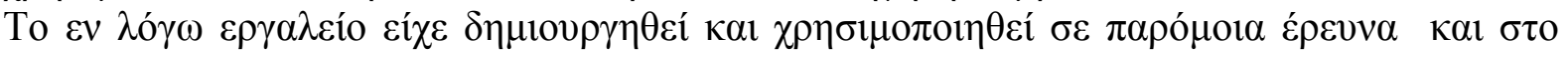

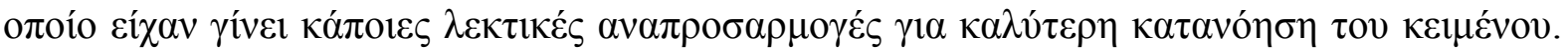

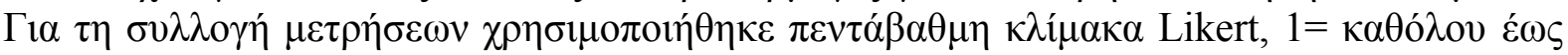

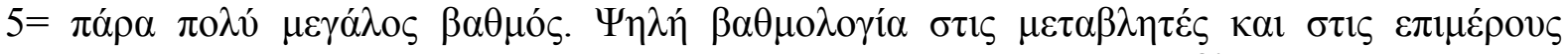

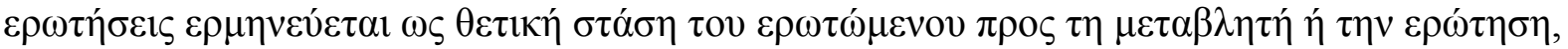

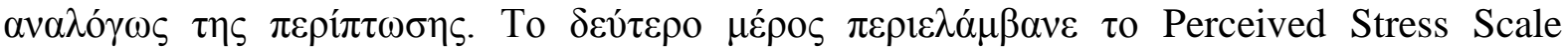




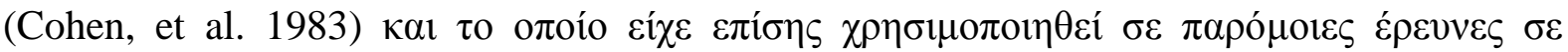

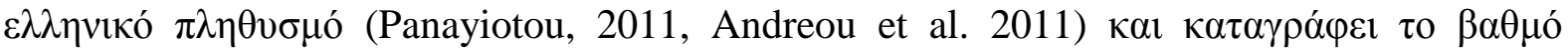

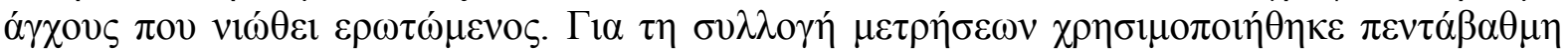

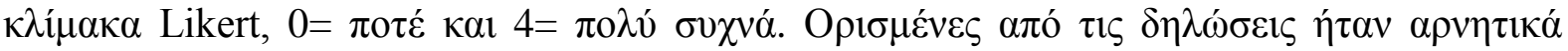

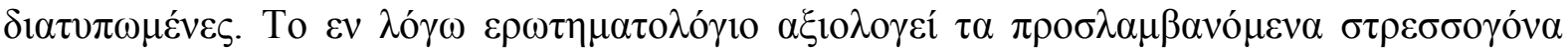

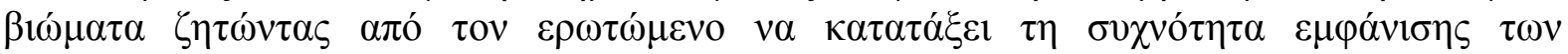

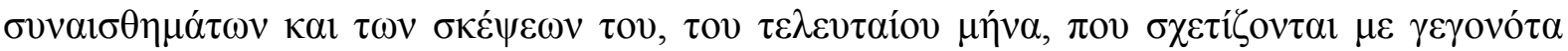

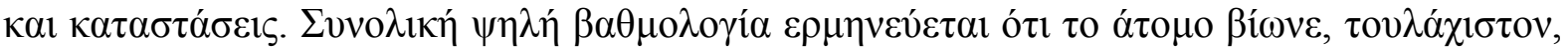

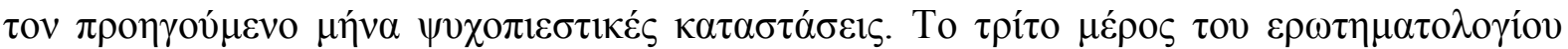

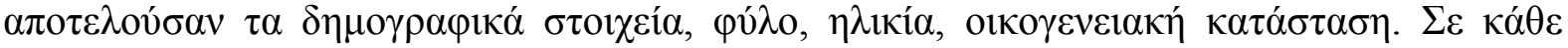

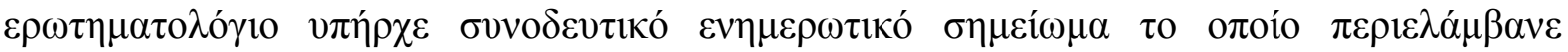

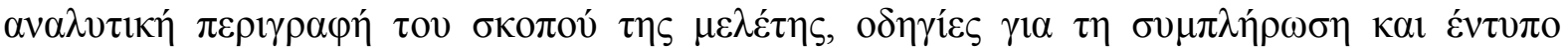

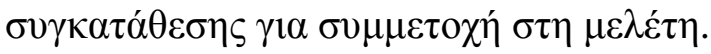

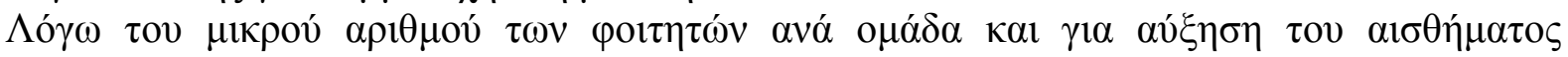

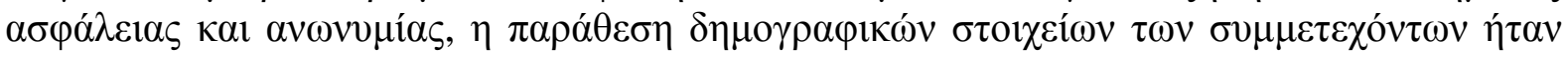
$\pi \varepsilon \rho 10 \rho ı \mu \varepsilon^{\prime} \eta \eta$.

\section{А $\pi 0 \tau \varepsilon \lambda \varepsilon ́ \sigma \mu \alpha \tau \alpha$}

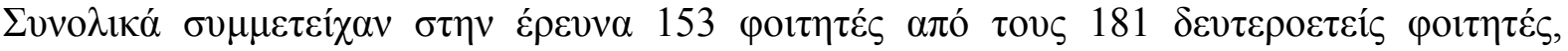

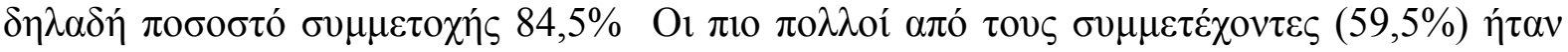

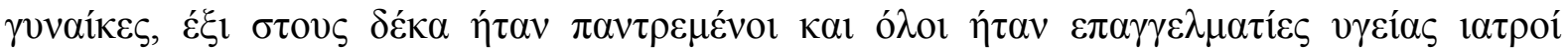

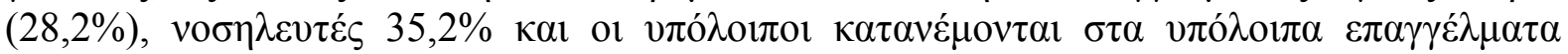

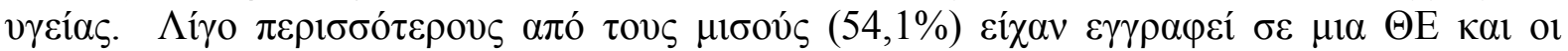

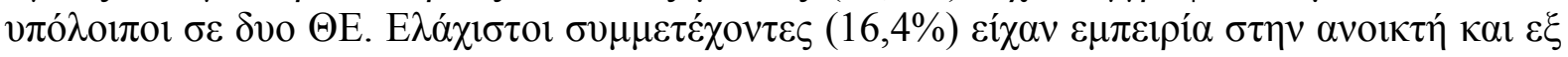

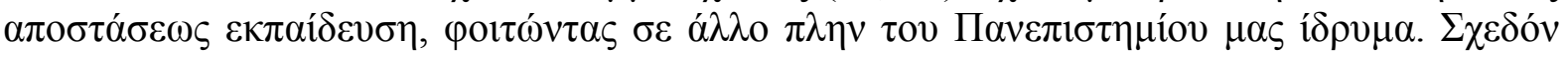

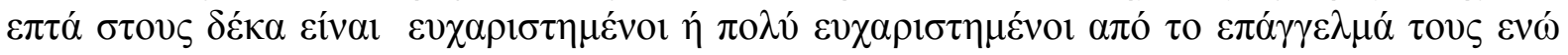

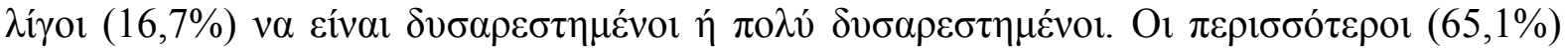

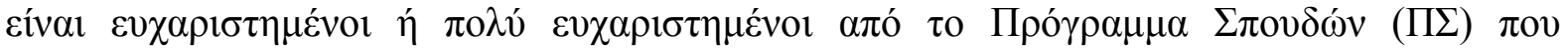

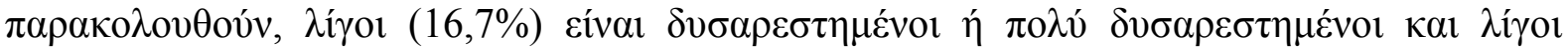

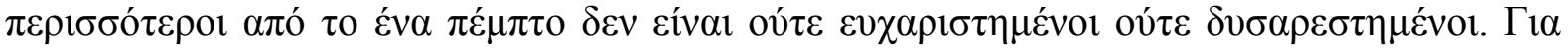

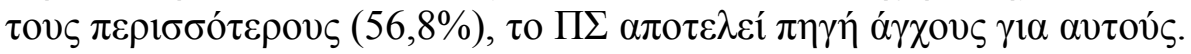

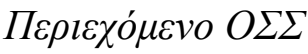

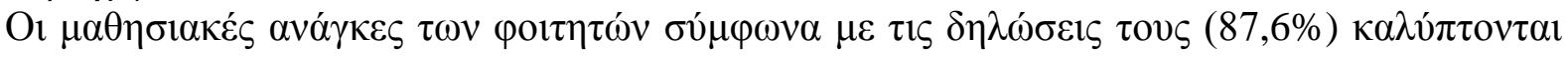

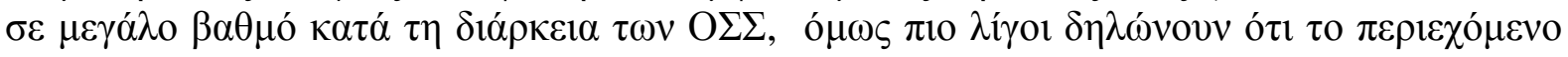

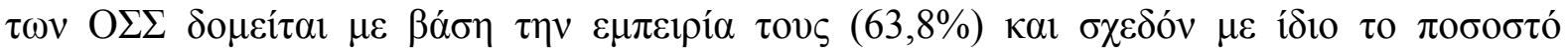

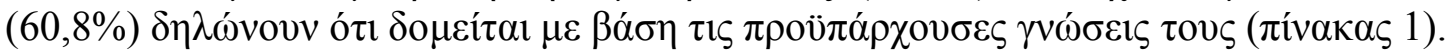

Пívaкas 1.

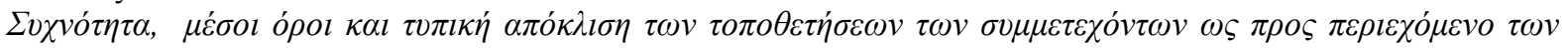
$O \Sigma \Sigma$

$\Delta \eta \lambda \omega ́ \sigma \varepsilon 1 \varsigma$

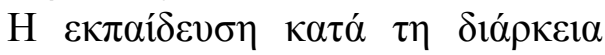

$\tau \omega \nu \quad \mathrm{O} \Sigma \Sigma \quad \beta \alpha \sigma i \zeta \varepsilon \tau \alpha l$ $\sigma \tau 1 \zeta$ $\mu \alpha \theta \eta \sigma 1 \alpha \kappa \varepsilon ́ \varsigma \quad \sigma \alpha \varsigma \quad \alpha v \alpha ́ \gamma \kappa \varepsilon \zeta \quad \kappa \alpha 1$
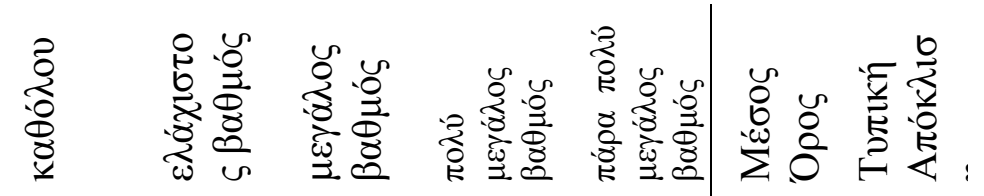
$\varepsilon v \delta 1 \alpha \varphi \varepsilon ́ p o v \tau \alpha$

$$
\%
$$

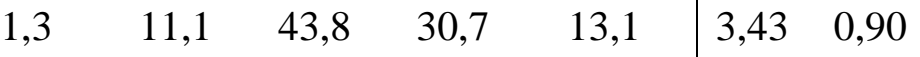




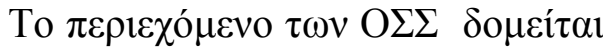
$\mu \varepsilon \beta \alpha ́ \sigma \eta \tau \eta v \varepsilon \mu \pi \varepsilon i \rho i ́ \alpha \quad \sigma \alpha \varsigma$

4

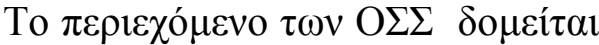

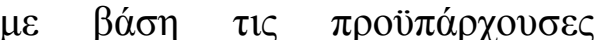

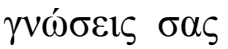

O1 $\varepsilon \pi i ́ \kappa \varepsilon v \tau \rho о \quad \tau \eta \varsigma \quad \varepsilon \kappa \pi \alpha 1 \delta \varepsilon v \tau \iota \kappa \eta$

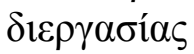

$\Lambda \alpha \mu \beta a ́ v o v \tau \alpha$ l

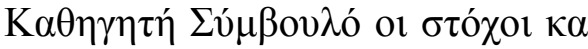

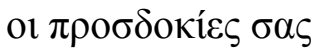

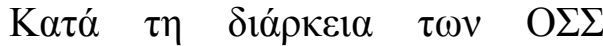

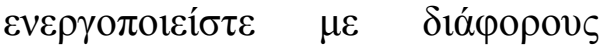

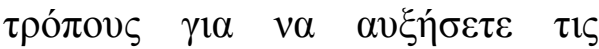

\section{0}

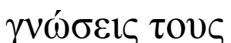

To $\pi \varepsilon p 1 \varepsilon \chi 0 ́ \mu \varepsilon v o \quad \tau \omega \nu \quad \mathrm{O} \Sigma \Sigma$

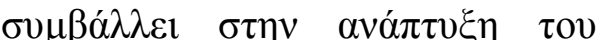

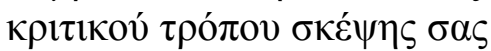

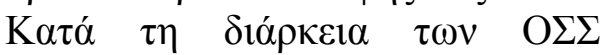

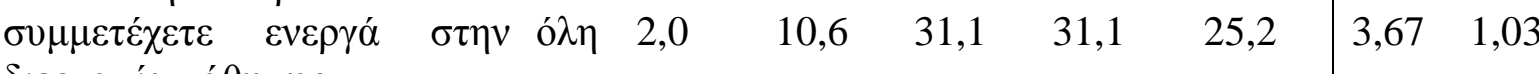

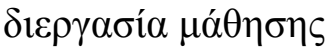

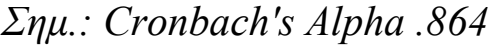

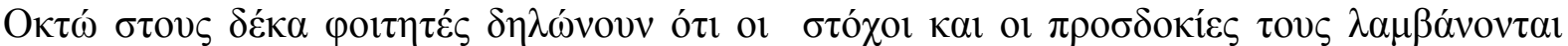

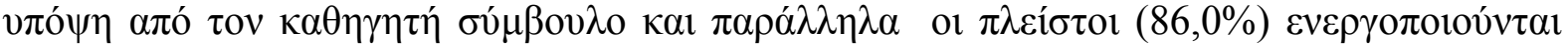

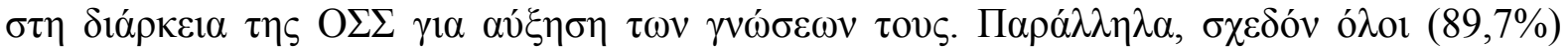

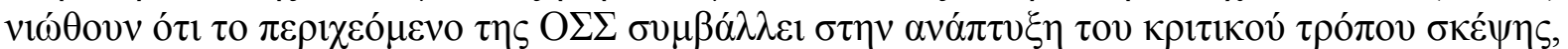

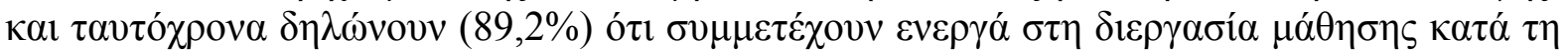

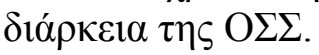

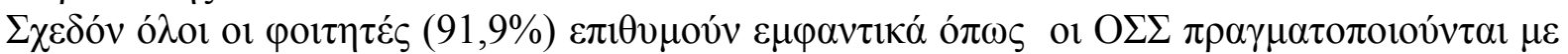

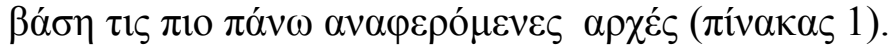

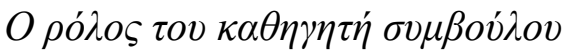

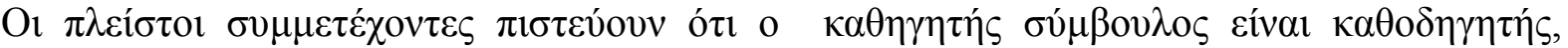

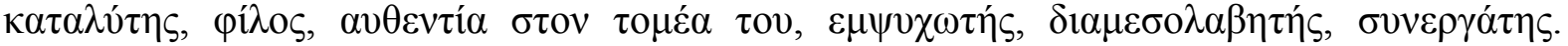

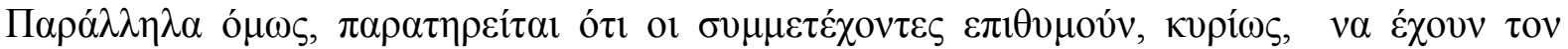

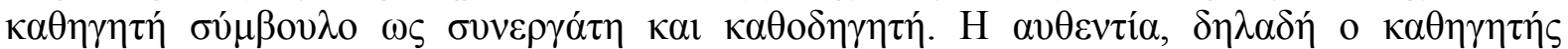

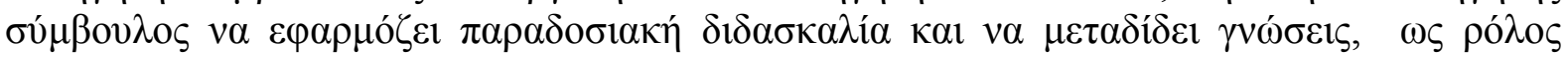

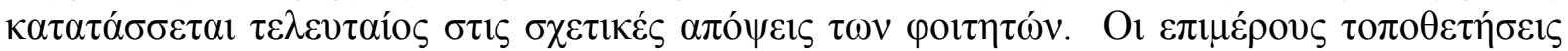

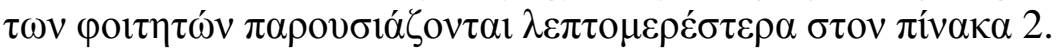

Пívakas 2.

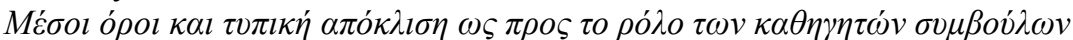

\begin{tabular}{|c|c|}
\hline $\mathrm{N}$ & 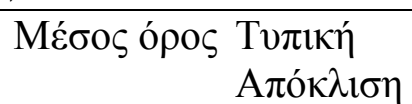 \\
\hline 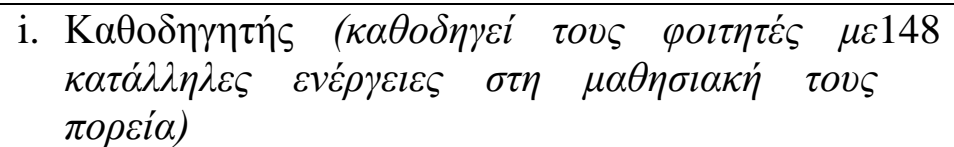 & .963 \\
\hline
\end{tabular}




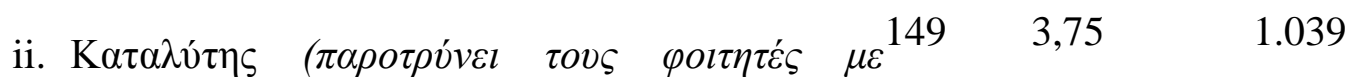
$\kappa \alpha \tau \alpha \dot{\lambda} \lambda \lambda \eta \lambda \alpha \kappa i v \eta \tau \rho \alpha)$

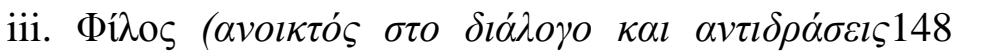

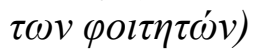

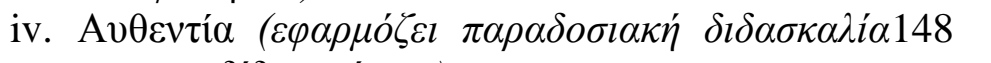
$\kappa \alpha \imath \mu \varepsilon \tau \alpha \delta i \delta \varepsilon \varepsilon l \gamma v \omega ́ \sigma \varepsilon l \varsigma)$

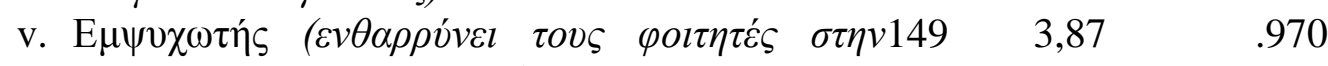

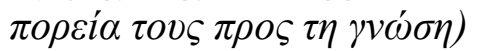

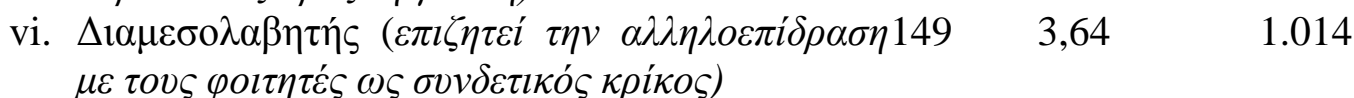

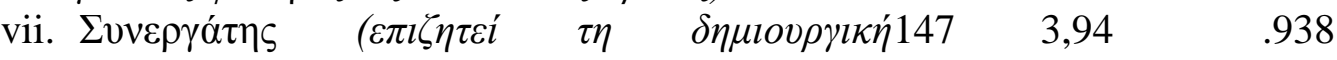

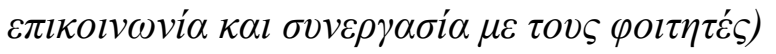

$\sum \eta \mu .:$ Cronbach's Alpha $=.875$

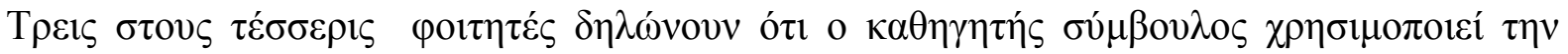

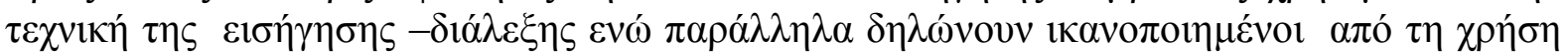

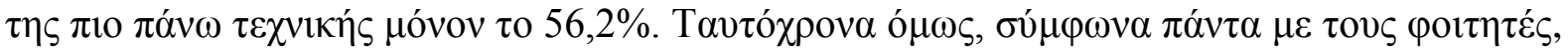

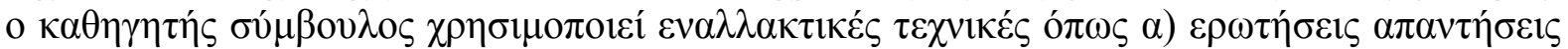

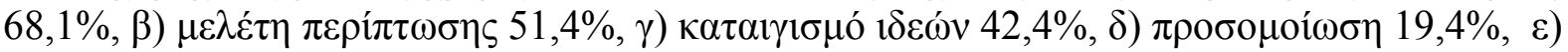

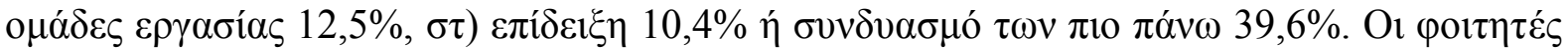

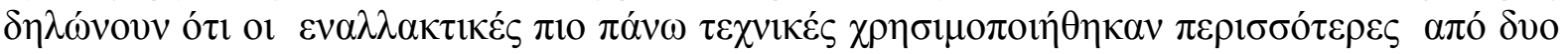

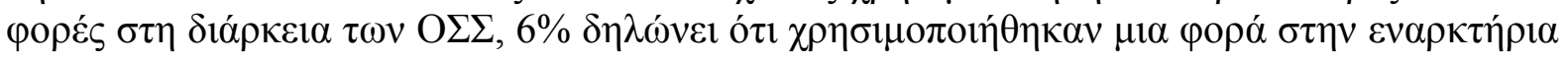

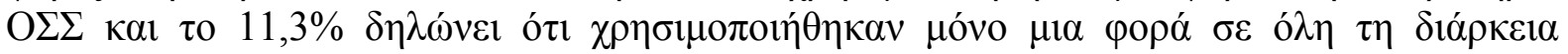

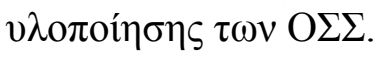

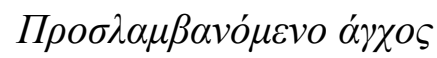

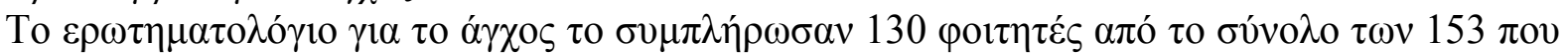

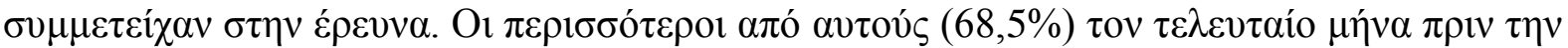

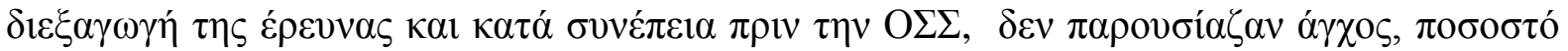

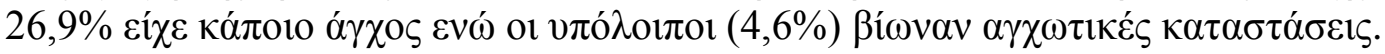

\section{$\Sigma v \zeta \eta ́ \eta \eta \sigma \eta-\Sigma v \mu \pi \varepsilon \rho \alpha ́ \sigma \mu \alpha \tau \alpha$}

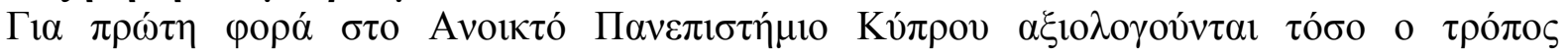

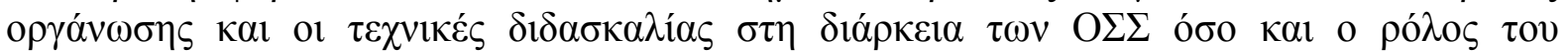

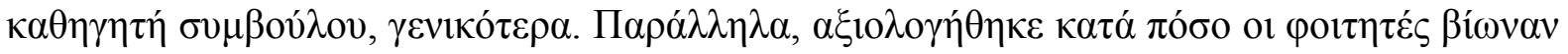

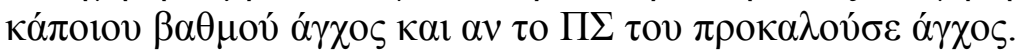

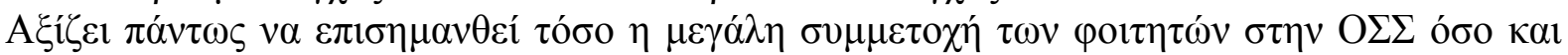

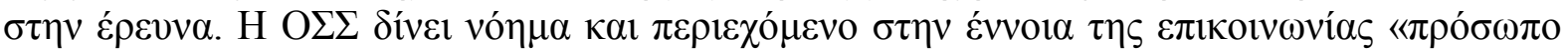

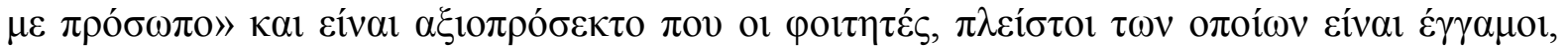

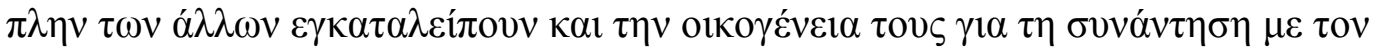

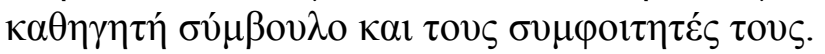

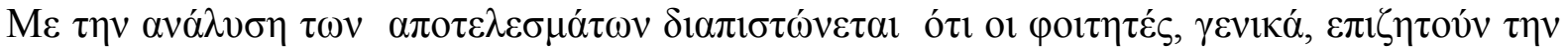

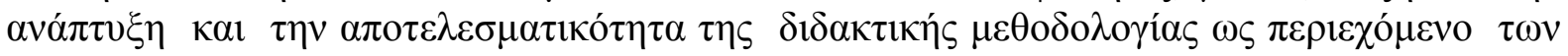

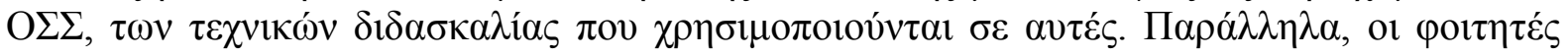

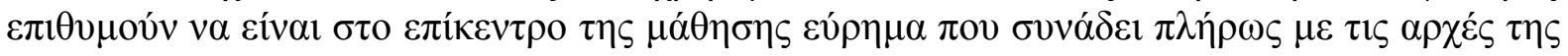

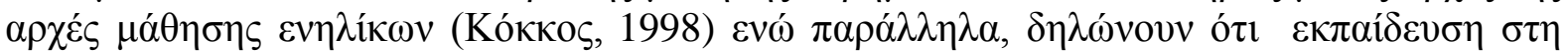

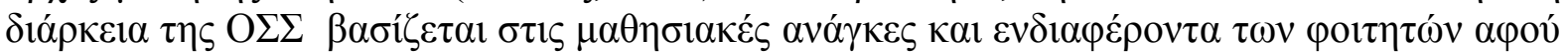

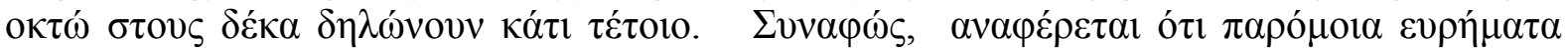

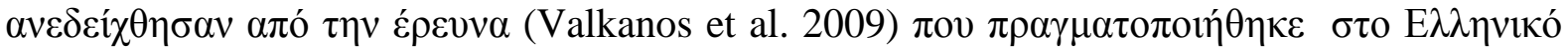




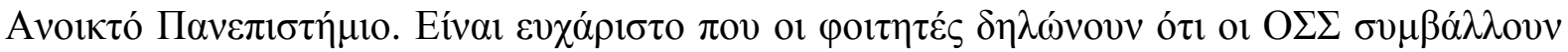

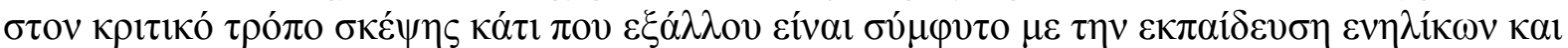
$\mu \varepsilon \tau \eta \nu \varepsilon \xi \alpha \pi \sigma \sigma \tau \alpha \dot{\sigma} \sigma \varepsilon \omega \varsigma \varepsilon \kappa \pi \alpha i ́ \delta \varepsilon v \sigma \eta$.

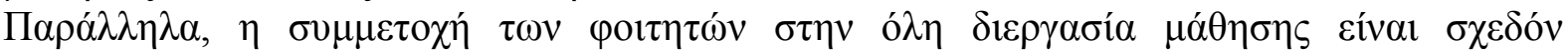

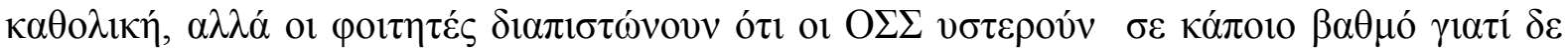

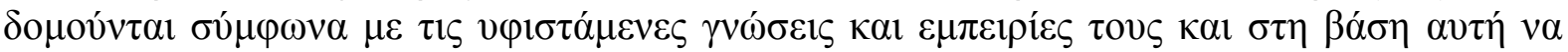

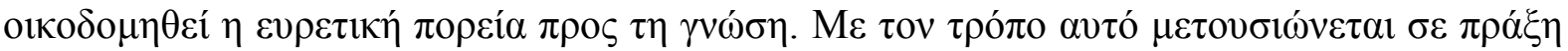

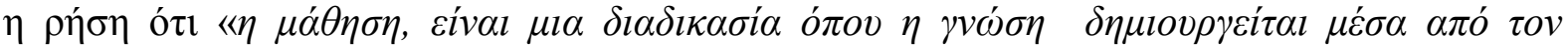

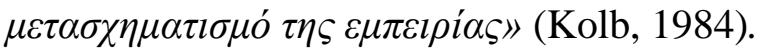

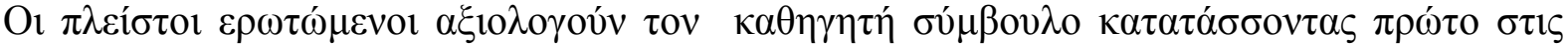

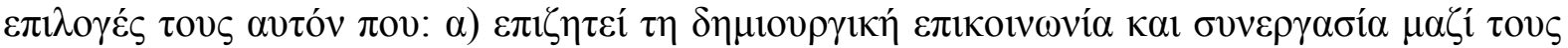

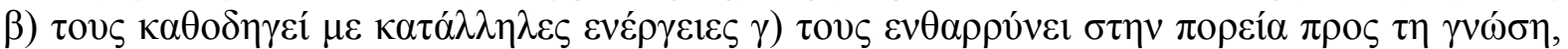

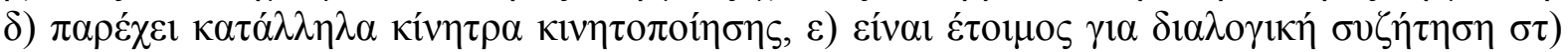

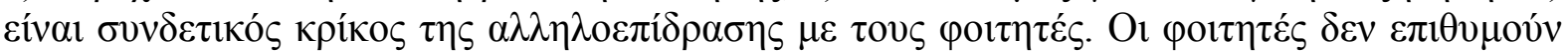

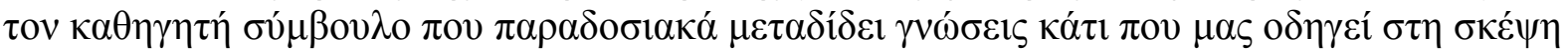

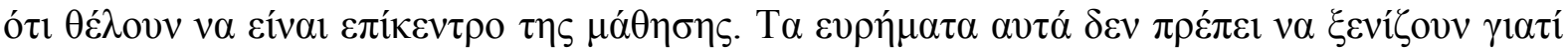

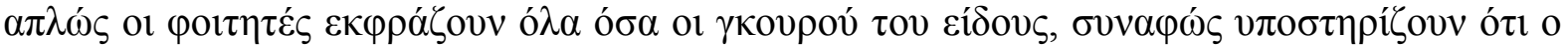

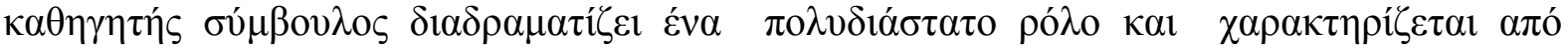

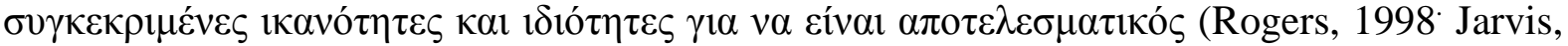
2007).

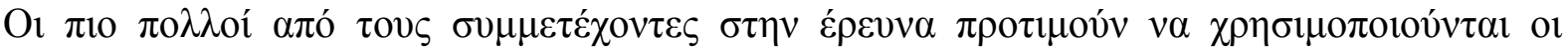

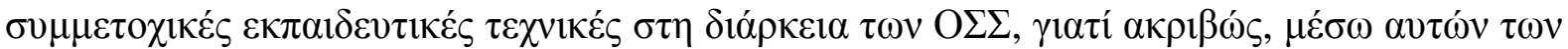

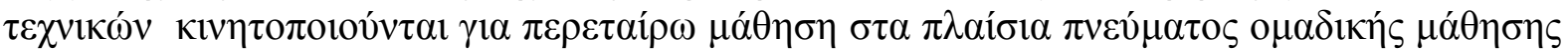

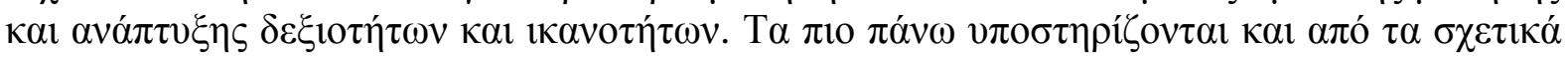
$\varepsilon v \rho \eta ́ \mu \alpha \tau \alpha \tau \omega v$ Fragoulis et al. (2008).

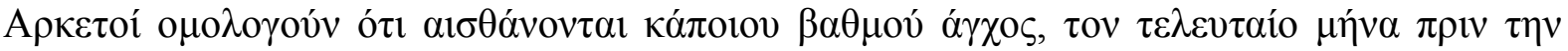

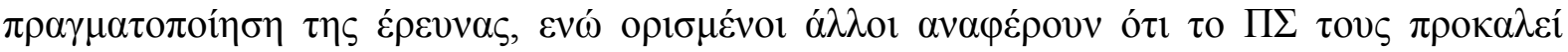

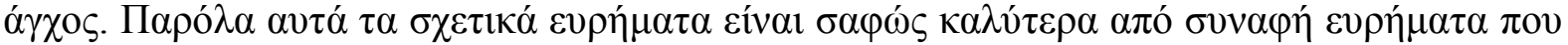

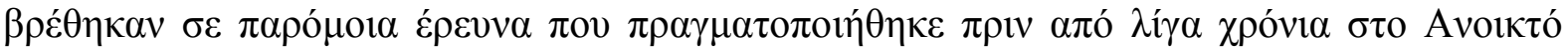

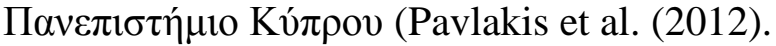

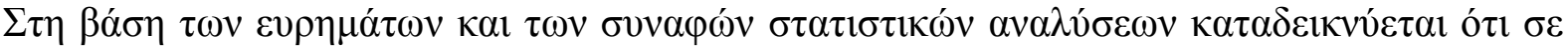

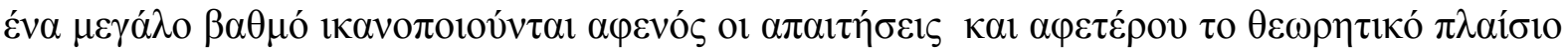

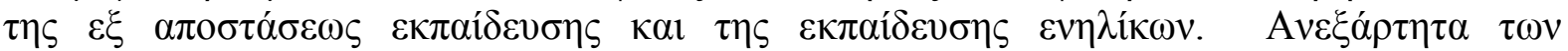

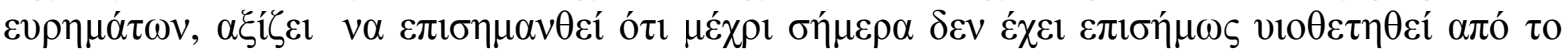

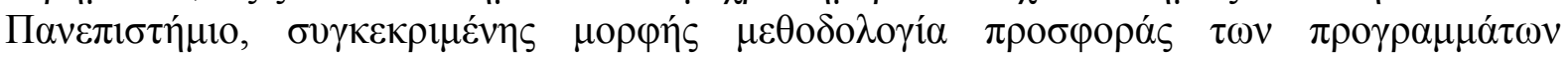

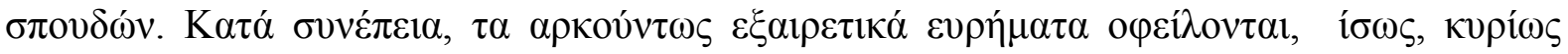

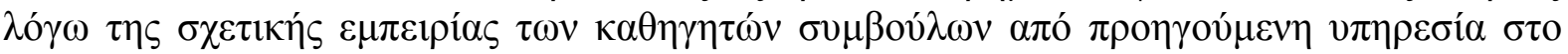

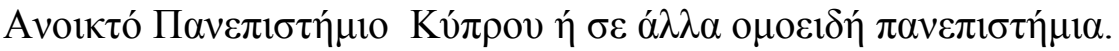

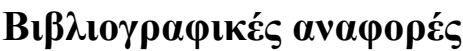

Andreou E., Alexopoulos E., Lionis C., Varvogli L., Gnardellis C., Chrousos G., and Darviri C. (2011). Perceived Stress Scale: Reliability and Validity Study in Greece. Int J Environ Res Public Health. 2011 August; 8(8): 3287-3298.

Cohen, S., Kamarck, T., and Mermelstein, R. (1983). A global measure of perceived stress. Journal of Health and Social Behavior, 24, $386-396$.

Jarvis, P. (2007). Adult and Continuing Education: Theory and Practice, $\sigma \varepsilon \lambda 235$. UK: Routledge.

Fragoulis, I., Papadakis, S. \& Fillips, N. (2008). The contribution of self-evaluation exercise in blended adult education. Training and Management Development Methods, 22, 45-63.

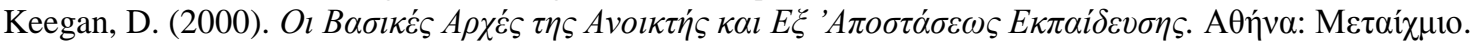

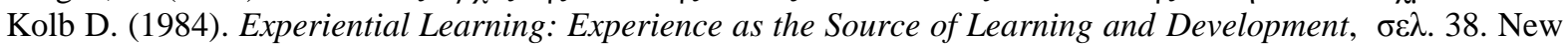
Jersey: Prentice- Hall, Enlgewood Cliffs. 
Modesto S.T. and Tau R. D. (2010). Introducing distance education, Virtual University for the Small States of

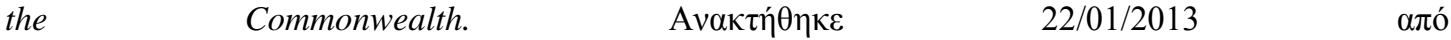
http://www.col.org/SiteCollectionDocuments/Introducing_Distance_Education.pdf.

Panayiotou G. (2011). Perceived stress, support, coping and health among university personnel: The role of experiential avoidance. Psychology \& Health, vol. 26, pp. 170-175.

Pavlakis, A. \& Kaitelidou, D. (2012). Burnout Syndrome in Students of a Distance Learning Program: The Open University of Cyprus Experience. European Journal of Open, Distance and E-Learning, ISSN 1027-5207, 2012, Issue 1, p. 10.

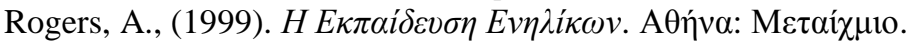

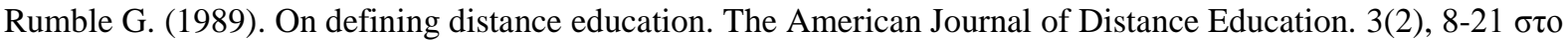

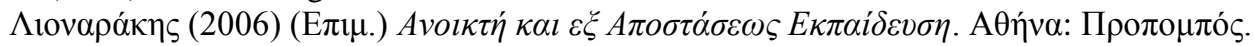

Valkanos E., Papavassiliou-Alexiou I., Fragoulis I. (2009). A Study on Perceptions of Open University Students about the Use of Effectiveness of Collaborative Teaching Methods during the Tutorial Group Meetings. Review of European Studies Vol. 1, No. 2.

Zembylas M., Theodorou M., Pavlakis A. (2008). The role of emotions in the experience of on line learning: challenges and opportunities. Educational Media International Vol 45, No.2 June, 107-117.

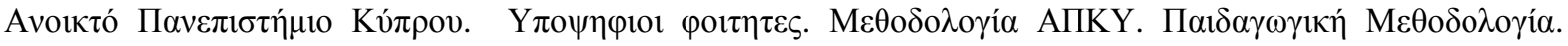

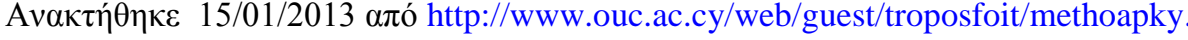

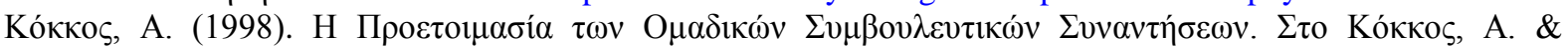

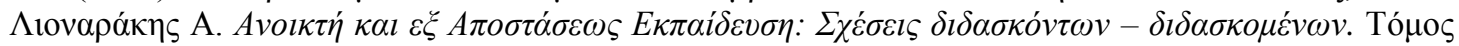

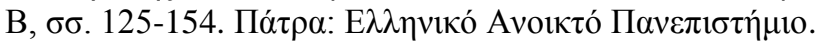

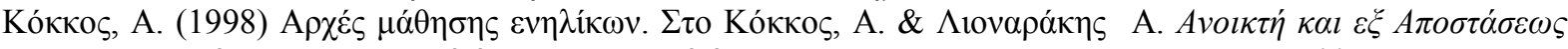

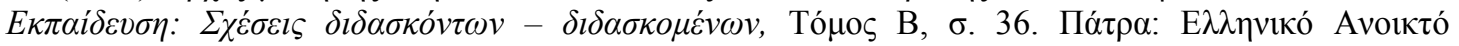

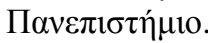

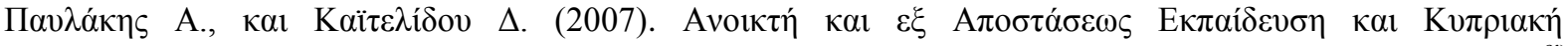

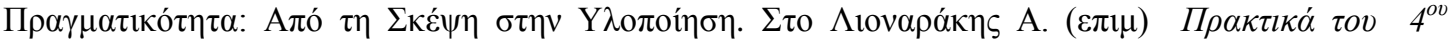

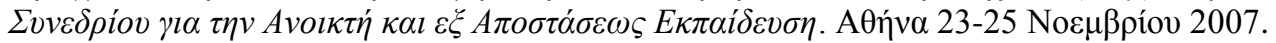

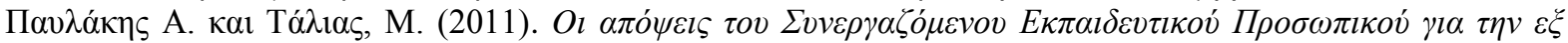

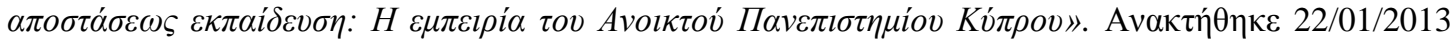
$\alpha \pi$ ó http://icodl.openet.gr/index.php/icodl/2011/schedConf/presentations.

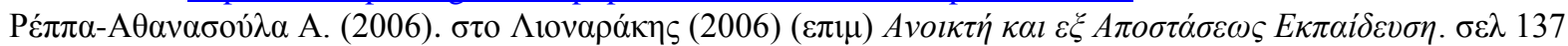

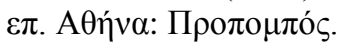

